\title{
THEOREMS OF NAMIOKA AND R. E. JOHNSON TYPE FOR UPPER SEMICONTINUOUS AND COMPACT VALUED SET-VALUED MAPPINGS
}

\author{
JENS PETER REUS CHRISTENSEN ${ }^{1}$
}

\begin{abstract}
Some recent results of Namioka on strong continuity of weakly continuous mappings (in a dense $G_{\delta}$ set) and results of R. E. Johnson on norm separability of the range of such mappings (under conditions on the domain space) are shown to have analogues for upper semicontinuous and compact valued set-valued mappings. Some substantial improvements of known automatic continuity results for such mappings are obtained.
\end{abstract}

0. Introduction. Let $F: X \rightarrow Y$ be a correspondence (set-valued mapping) from the Hausdorff topological space $X$ to the Hausdorff topological space $Y$. In the present paper the value $F(x)$ will always be a closed set (maybe empty and often compact) in $Y$.

The graph $\operatorname{Gr}(F)$ of the correspondence $F$ is the set

$$
\operatorname{Gr}(F)=\{(x, y) \in X \times Y: y \in F(x)\} .
$$

Suppose that $\operatorname{Gr}(F)$ is closed and $F(x)$ is a nonempty compact subset of $Y$ for each $x \in X$. By Zorn's lemma any such correspondence contains a correspondence $\tilde{F} \subseteq F$ with the same properties and being minimal (w.r.t. inclusion) in the set of all correspondences with closed graph and nonempty compact values. From now on minimal for a correspondence always refer to the above-mentioned minimality. In particular minimal correspondences have nonempty values.

Intuitively $\tilde{F}$ ought to be "close" to singlepoint-values and continuous at most points (since taking a minimal subcorrespondence should be analogous to taking a continuous selection). We shall show that under some assumptions on $X$ (gametheoretical) and a continuity assumption on the original correspondence $F$ (usco: upper semicontinuous and compact valued; from now on the term usco will be used in that sense. The meaning of upper semicontinuity will be explained below), we have that $\tilde{F}$ is singlepoint-valued and norm continuous at a dense $G_{\delta}$ subset $A$ of $X$, if $Y$ is a Banach space with weak topology. Also, under an additional countable chain condition on the domain space $X, \tilde{F}$ will automatically have norm separable

Received by the editors November 9, 1981 and, in revised form, February 3, 1982.

1980 Mathematics Subject Classification. Primary 54C60.

Key words and phrases. Automatic continuity in a dense $G_{\delta}$ set, nonlinear automatic continuity, strong continuity of usco set-valued mappings.

'The author was supported by the Danish Natural Science Research Council while completing this work. 
range. The results improve previous results of this type (see [ 2 and 3]) for singlepoint-valued mappings. The proofs are nontrivial modifications of the methods employed in the above-mentioned papers.

By an example we show that even if $X$ is a locally compact metric space with a countable base and $Y$ is compact, a minimal usco correspondence of the type described above need not be singlepoint-valued at any point, if the space $Y$ is too "large".

1. Preliminaries. The class of Hausdorff spaces $X$, which may serve as domain spaces in the present paper, is the class of spaces favorable for the player $\alpha$ in a topological "game" described below.

Let us consider the following "game" played on the Hausdorff space $X$ by the player $\alpha$ and the player $\beta$. The player $\beta$ starts by choosing a nonempty open subset $U_{1}$ of the Hausdorff topological space $X$. Then the player $\alpha$ chooses an open subset $V_{1}$ of $U_{1}$ and a point $x_{1} \in V_{1}$ (his move is the pair $\left(V_{1}, x_{1}\right)$ with $\left.x_{1} \in V_{1} \subseteq U_{1}\right)$. Then $\beta$ chooses an open nonempty subset $U_{2}$ of $V_{1}$ (she may choose as desired, but is expected to try to "escape" the point chosen by $\alpha$ ).

Next $\alpha$ chooses an open subset $V_{2}$ of $U_{2}$ and $x_{2} \in V_{2} \cdots$. We shall fix the rule that $\alpha$ wins, iff any subsequence $X_{n_{p}}$ of the sequence $x_{n}$ accumulates to at least one point of the set

$$
I=\bigcap_{n=1}^{\infty} V_{n}=\bigcap_{n=1}^{\infty} U_{n} .
$$

We permit only strategies for $\alpha$ depending on the previous move by $\beta$. A strategy for $\alpha$ is therefore a mapping $s: V \rightarrow V^{*}$ of the set of nonempty open sets $V$ into the set $V^{*}$ of pairs $(U, x)$ with $x \in U \in V$ and such that for $s(U)=\left(s_{1}(U), s_{2}(U)\right)$ we have $s_{2}(U) \in s_{1}(U) \subseteq U$ for all $U \in V$. We shall say that $s$ is a winning strategy (for $\alpha$ ), iff $\alpha$ wins irrespective of the behaviour of $\beta$, provided $\alpha$ moves according to $s$.

We shall call $X$ a $\sigma$-well $\alpha$-favorable space, iff a winning strategy exists for $\alpha$ in the $\sigma$-game described above (the closely related class of $\tau$-well $\alpha$-favorable spaces is obtained by replacing sequences with nets). We refer to [1 and 2] for discussion of this game.

\section{Formulation and proofs of the main results.}

Definition. The correspondence $F: X \rightarrow Y$ from the Hausdorff space $X$ to the Hausdorff space $Y$ is usco (upper semicontinuous and compact valued) if $F(x) \subseteq Y$ is compact for all $x \in X$ and for all open sets $U$ in $Y$ the set

$$
\{x \in X: F(x) \subseteq U\}
$$

is open in $X$.

It is easy to show that the graph of an usco correspondence is automatically closed, but having a closed graph is unfortunately not sufficient for being usco.

Also we note, that if $F: X \rightarrow Y$ is an usco correspondence from the Hausdorff space $X$ to the Hausdorff space $Y$ then any correspondence with closed graph contained in the graph of $F$ is automatically usco. A special case of this observation is the fact that closed graph implies usco, if only the range is contained in a compact 
set. Since the last observation above is important to our paper and we have been unable to find a reference, we shall sketch a proof.

Let $\tilde{F}: X \rightarrow Y$ be a correspondence with closed graph contained in the graph of the usco correspondence $F: X \rightarrow Y$. Suppose (to derive a contradiction) that $\tilde{F}$ is not upper semicontinuous. Then there is a point $x_{0} \in X$ such that $\tilde{F}$ is not upper semicontinuous at $x_{0}$ (the meaning of this term should be obvious). Let $U \subseteq Y$ be an open set with $\tilde{F}\left(x_{0}\right) \subseteq U$ and let $x_{d} \rightarrow x_{0}(d \in D)$ be a net in $X$ with the property that there exists $y_{d} \in \tilde{F}\left(x_{d}\right) \backslash U$ (negation of semicontinuity at $x_{0}$ ). The upper semicontinuity of $F$ implies that the net $y_{d}(d \in D)$ accumulates to some point $y_{0}$ of $F\left(x_{0}\right)$ (of course the compactness of $F\left(x_{0}\right)$ is essential for this argument). Hence the net $\left(x_{d}, y_{d}\right)$ accumulates to $\left(x_{0}, y_{0}\right)$ and we get a contradiction with the assumed fact that the graph of $\tilde{F}$ is closed. This concludes the sketch of the proof.

The reader would probably think that we have not used the full strength of $\sigma$-well $\alpha$-favorability in the proof of our Theorem 1 below. This is formally true. However, Flemming Topsøe pointed out to the author that in the definition of $\sigma$-well $\alpha$-favorability one need only assume that the original sequence $x_{n}$ has an accumulation point in $I$. Then to see that $x_{n_{p}}$ has an accumulation point in $I$ we consider a "game" where $\beta$ in her move number $p-1(p \geqslant 2)$ chooses $U_{n_{p}-1}$.

THEOREM 1. Let $F: X \rightarrow B$ be a usco correspondence with nonempty values from the $\sigma$-well $\alpha$-favorable space $X$ into the Banach space $B$ with weak topology. Let $\tilde{F} \subseteq F$ be minimal (by inclusion) among all correspondences with closed graph and nonempty compact values (whose graph is contained in $\operatorname{Gr}(F)$ ). Then there is a dense $G_{\delta}$ set $A \subseteq X$ such that the following condition (*) holds in each point $x_{0} \in A$ :

(*) The compact set $\tilde{F}\left(x_{0}\right)$ consists of a single point and for all $e>0$ the set $\{x \in X$ : $\left.\sup \left\{\left\|y-\tilde{F}\left(x_{0}\right)\right\|: y \in \tilde{F}(x)\right\}<e\right\}$ is a neighbourhood of $x_{0}$ in $X(\|\cdot\|$ is the norm in $B)$.

Proof. The theorem is of course analogous to the improved version of Namioka's theorem [1, Theorem 1], and the proof is indeed an adaption. However, this adaption is not completely trivial.

First we remark that $\tilde{F}$ is also usco and it is no restriction to assume (as we shall henceforth do) that $F$ is already minimal. We define the local oscillation $\alpha_{F}(x)$ of $F$ at the point $x \in X$ by the expression

$$
\alpha_{F}(x)=\inf \left\{\sup \left\{\left\|y_{1}-y_{2}\right\|: y_{1} \in F\left(x_{1}\right), y_{2} \in F\left(x_{2}\right), x_{1}, x_{2} \in U \in V(x)\right\}\right\}
$$

where the infimum is taken over all neighbourhoods of $x$. Since $\alpha_{F}$ is obviously upper semicontinuous the set $A=\left\{x: \alpha_{F}(x)=0\right\}$ is a $G_{\delta}$ set. It is easy to see that $A$ is exactly those points in which the continuity condition (*) holds. Now the space $X$ is Baire. Therefore, if $A$ is not dense in $X$, then there exists $k>0$ such that $\{x$ : $\left.\alpha_{F}(x)<k\right\}$ is not dense, i.e. $\alpha_{F}(x) \geqslant k>0$ on a nonempty open set $U$.

Replacing $U$, which is $\sigma$-well $\alpha$-favorable, with $X$, we have reduced the whole proof of Theorem 1 to the derivation of a contradiction from the assumption that $\alpha_{F}(x) \geqslant k>0$ for all $x \in X$. Let us make this assumption and derive a contradiction (the restriction to an open subset of a minimal usco correspondence is easily shown to be minimal). 
Let us start the topological "game" between the players $\beta$ and $\alpha$. Naturally $\alpha$ chooses to move according to some winning strategy $s$. The player $\beta$ chooses the following strategy:

In her first move $\beta$ chooses her set to be the whole space $X$. Assume that the moves

$$
U_{1}, s\left(U_{1}\right)=\left(V_{1}, x_{1}\right), \ldots, U_{n-1}, s\left(U_{n-1}\right)=\left(V_{n-1}, x_{n-1}\right)
$$

have been made with $n \geqslant 2$. In the move number $n$ for $\beta$, the compact set

$$
H_{n}=\operatorname{conv}\left(\left\{y_{1}, \ldots, y_{n-1}\right\}\right)
$$

is considered, where $y_{i} \in F\left(x_{i}\right)$ for $i=1, \ldots, n-1$ are arbitrarily selected points.

Let us define the set

$$
\tilde{H}_{n}=\left\{b \in B: \delta\left(b, H_{n}\right) \leqslant k / 3\right\}
$$

(the symbol $\delta$ is used for the metric on $B$ induced by the norm $\|\cdot\|$ ).

Let us consider the closed set in $X$ defined by

$$
C_{n}=\left\{x \in X: F(x) \cap \tilde{H}_{n} \neq \varnothing\right\} .
$$

That $C_{n}$ is indeed closed follows from the fact that $\tilde{H}_{n}$ is weakly closed and $F$ is usco. We intend to show that $C_{n}$ has empty interior!

Suppose this is not the case. We may cover $H_{n}$ with finitely many closed balls with center in $H_{n}$ and radius $\frac{5}{12} k$ (say $B_{1}, \ldots, B_{p}$ ). The closed sets

$$
\left\{x \in X: F(x) \cap B_{i} \neq \varnothing\right\}, \quad i=1, \ldots, p,
$$

form a finite covering of $C_{n}$. Hence one of them (say the first) has nonempty interior. Thus there exists a nonempty open subset $U$ of $X$ with $F(x) \cap B_{1} \neq \varnothing$ for all $x \in U$. We note that the restriction of a minimal usco correspondence to an open set is also minimal. Hence $F$ coincides on $U$ with $F(x) \cap B_{1}$, which is also usco being contained in an usco correspondence and having closed graph (and whose values are nonempty!). But this implies that the local oscillation $\alpha_{F}$ in each point of $U$ is at most $\frac{10}{12} k$. This contradiction shows that indeed $C_{n}$ has empty interior. Our player $\beta$ thus may choose her set $U_{n}$ to be disjoint from the closed set $C_{n}$, and we shall assume that she does so.

We use the fact that $\alpha$ is winning the "game" to choose $x_{\infty} \in \cap_{n=1}^{\infty} V_{n}=\bigcap_{n=1}^{\infty} U_{n}$ such that $x_{n}$ (the sequence of points $\alpha$ chosen by using his winning strategy $s$ ) accumulates to $x_{\infty}$. The sequence $y_{n} \in F\left(x_{n}\right)$ accumulates weakly to some element $y_{\infty} \in F\left(x_{\infty}\right)$ (an easy consequence of the usco property, of course $y_{\infty}$ is not uniquely determined). Hence (the Hahn-Banach theorem) suitable convex combinations of the $y_{n}$ 's tends uniformly (in norm) to $y_{\infty}$. But the distance of $y_{\infty}$ to any convex combination of the $y_{n}$ 's is at least $k / 3$ as is immediately seen from the strategy of $\beta$ described above. This last contradiction concludes the proof of Theorem 1.

It does not follow from the argument that $F$ has automatically the continuity property (*) in each point, in which $F$ is singlepoint-valued. This is of course too much to hope, since it is not true even if $F$ is globally singlepoint-valued. If it could be shown that for the correspondence $F$ (with the conditions of Theorem 1) there exists a dense $G_{\delta}$ set $A$ in $X$ such that for $x \in A$ the set of $y \in F(x)$ being the (*) 
point of some minimal usco correspondence contained in $F$ were norm (weakly) dense in $F(x)$, then of course $F$ would be norm (weakly) lower semicontinuous at each point of $A$. We have not been able to decide this question.

The continuity property "Kenderov continuity" defined below is inspired of Petar Kenderov's results in [4]. But the continuity statement is much stronger (although still weak) than the condition called (cp) in [4]. Furthermore, we drop completely the requirement that the values of the correspondence in question be convex and extend vastly the allowed class of domain spaces. But since Kenderov permits other spaces than metrizable locally convex vector spaces with weak topology (the only spaces for which our methods seem to work), his results are not completely contained in ours. With this qualification our Theorem 2 below is an improvement of a result of Petar Kenderov in [4].

THEOREM 2. Let $F: X \rightarrow B$ be a usco correspondence with all assumptions of Theorem 1 (except minimality). Then there is a dense $G_{\delta}$ set $A \subseteq X$ such that $F$ is "Kenderov continuous" at each point $x \in A$ in the following sense:

There exists $y \in F(x)$ such that for each $e>0$ there corresponds a neighbourhood $U$ of $x$ with

$$
\operatorname{dist}\left(y, F\left(x^{\prime}\right)\right)=\inf \left\{\left\|y^{\prime}-y\right\|: y^{\prime} \in F\left(x^{\prime}\right)\right\}<e
$$

whenever $x^{\prime} \in U$.

Proof. We choose a minimal usco correspondence contained in $F$ and apply Theorem 1. This concludes the proof.

Perhaps surprisingly it turns out that minimal usco correspondences of the type considered in Theorem 1 is so close to single valued, that a result on norm separability of the range extends to such correspondences. Theorem 3 below extends an extension of a result due to R. E. Johnson (see [2 and 3]) to minimal usco correspondences whose domain space is $\sigma$-well $\alpha$-favorable and has a countable chain condition on the open sets.

THEOREM 3. Let $F: X \rightarrow B$ be a minimal usco correspondence with all the assumptions of Theorem 1 and assume that the domain space $X$ has a countable chain condition on the open sets. Then the range of $F$ defined by

$$
\operatorname{range}(F)=\bigcup\{F(x): x \in X\}
$$

is a norm separable subset of the Banach space $B$.

Proof. The idea of the proof is very similar to the proof of Theorem 2 in [2]. For the sake of completeness we give the whole argument. For each $n$ we choose a maximal family $O_{i}^{n}\left(i \in I_{n}\right)$ of pairwise disjoint open subsets of $X$ with $\operatorname{diam}\left(F\left(O_{i}^{n}\right)\right)$ $\leqslant 1 / n$ for $F\left(O_{i}^{n}\right)=\bigcup\left\{F(x): x \in O_{i}^{n}\right\}$. Let us observe that $G_{n}=\bigcup\left\{O_{i}^{n}: i \in I_{n}\right\}$ is dense in $X$ because of maximality (and Theorem 1!). The countable chain condition ensures that each $I_{n}$ is at most countable. Let $b_{n} \in B$ be a sequence such that each of the sets $F\left(O_{i}^{n}\right)$ contains at least one $b_{n}$. Let $C=\operatorname{cl} \operatorname{conv}\left(\left\{b_{n}\right\}\right)$, since the weak and the norm closure of a convex set coincides, we see that $C$ is a weakly closed norm 
separable set containing each $b_{n}$. Let $C_{n}$ be the weakly closed set

$$
C_{n}=\{b \in B: \delta(b, C) \leqslant 1 / n\} .
$$

The closed set $\left\{x \in X: F(x) \cap C_{n} \neq \varnothing\right\}$ contains $G_{n}$ and is therefore equal to $X$. The minimality of $F$ now ensures that the whole range of $F$ is indeed contained in $C_{n}$. This being true for each $n$ the proof of Theorem 3 is finished.

In all proofs above we might let $B$ be a metrizable locally convex topological vector space with weak topology and the norm be some translation invariant metric whose balls are convex (the metric generating the topology of course). Then all proofs work fine.

We might also permit the range space to be just some metric space $(Z, d)$. To see this we discuss the imbedding of $(Z, d)$ into a Banach space discussed in the papers [1 and 2]. Let $K$ be

$$
K=\left\{f: Z \rightarrow[-1,1]:\left|f\left(z_{1}\right)-f\left(z_{2}\right)\right| \leqslant d\left(z_{1}, z_{2}\right) \text { for all } z_{1}, z_{2} \in Z\right\} .
$$

Then of course $K$ is a compact Hausdorff space with the topology of pointwise convergence. Let $\theta(z)$ be the continuous function on $K$ defined by $\theta(z)(k)=k(z)$. Then $\theta$ is uniformly continuous in both directions (if $\operatorname{diam}(Z)<1, \theta$ is even an isometry) with respect to sup norm on $C(K)$. This imbedding is also a homeomorphism with respect to the weak topology on the Banach space $C(K)$ of continuous functions on $K$ (restricted to the image $\theta(Z)$ ).

Using the same arguments as in [1 and 2] it is now not difficult to show that we might even permit as range space a space of continuous functions from some compact Hausdorff space into some metric space equipped with the topology of pointwise convergence. The continuity and density statements would be with respect to the sup metric on this function space.

With those observations in mind it might be of interest to note that even for locally compact separable metric domain spaces one cannot obtain good results (of the above type), if the range space is compact but too "large".

3. A counterexample. The example given below is a slight modification of a function used also in [1] and communicated orally to the author by J. HoffmannJørgensen. Let $X=Y=$ ]-1, 1[ be the open interval from -1 to 1 with its usual topology (induced by the euclidean metric). Let $Z$ be the space of mappings from $[-1,1]^{2}$ into $[-1,1]$ equipped with the topology of pointwise convergence. Thus $Z$ is a rather "large" compact Hausdorff space. As in [1] we define the function $F$ from $X \times Y$ into $Z$ giving the value of the function $F(x, y)$ at the point $(a, b)$ by

$$
F(x, y)(a, b)=2(x-a)(y-b) /\left((x-a)^{2}+(y-b)^{2}\right)
$$

(if this quotient is undefined, we put it equal to zero). As in [1] we see that $F$ is not continuous in any point of $X \times Y$, although $F$ is indeed separately continuous. Let $H$ be the usco correspondence from $X \times Y$ to $Z$ whose graph is the closure of the graph of $F$. 
THEOREM 4. The correspondence $H$ from $X \times Y$ to $Z$ defined above is minimal usco, and for $(x, y) \in X \times Y$ the set $H(x, y)$ is given by $H(x, y)=\{f:(a, b) \rightarrow[-1,1]$ : $f(a, b)=2(x, a)(y-b) /\left((x-a)^{2}+(y-b)^{2}\right)$ for $(a, b) \neq(x, y)$, in $(x, y) f$ may assume any value in $[-1,1]\}$.

The rather straightforward proof is left for the reader. We refer to [1, 4 and 5] for applications of the above results.

ACKNOWLEDGEMENTS. The author is indebted to J. Hoffmann-Jørgensen and Flemming Topsøe for stimulating and encouraging discussions.

\section{REFERENCES}

1. Jens Peter Reus Christensen, Joint continuity of separately continuous functions, Proc. Amer. Math. Soc. 82 (1981), 455-461.

2. L_ Remarks on Namioka spaces and R. E. Johnson's theorem on the norm separability of the range of certain mappings, Math. Scand. (to appear).

3. R. E. Johnson, Separate continuity and rneasurability, Proc. Amer. Math. Soc. 82 (1969), 420-422.

4. Petar Kenderov, Dense strong continuity of pointwise continuous mappings, Pacific J. Math. 89 (1980).

5. I. Namioka, Separate and joint continuity, Pacific J. Math. 51 (1974).

Kobenhavns Universitets Matematiske Institut, UniversitetsParken 5, 2100 KobenhaVn ø., DANMARK 